no diarrhoa, and (as I now learned from the father) the symptoms began soon after and not at dinner-time, I thougnt that if poison had been taken it was more probably an irritant than a corrosive, perhajs arsenic. I therefore removed in a carefully washed bottle part of the contents of the stomach, and rode direct home. I hastily prepared and applied the colour tests, without discovering any definite trace of poison." I also tried Reinsch's reduction process, and obtained a well-marked dark violet stain, the careful further examination of which to prove conclusively its exact character would have occupied more time than I thought the present condition of the patient warranted me in bestowing, especially as by this time the messenger who followed me had arrived from the patient's house. I explained to my brother the nature of the case, reminding him that the stain might or might not prove to be arsenic, and asked his advice and assistance. Upon seeing the patient and the cha racter of the matters vomited, he agreed with me upo the propriety of immediately using the stomach-pump, which was done, the viscus being carefully washed out with three or four pints of warm water until the fluid returned clear, a little sulphate of zinc being added at the commencement. The child was left comfortably dozing at about ten P.M. The hydrated sesquioxide of iron was prepared extemporaneously, and ordered to be given every half hour.

Next morning a messenger arrived, saying that the vomiting and pain had returned as severely as ever, and I was at some pains to have the lad removed to the intirmary. He lingered throngh this and the following day, and died on the morning of Friday, the 24th July.

Examination of the body discovered a round worm, ten or eleven inches long, in the large intestine, the existence of which during life had never been avowedly suspected, although the boy was seen by several medical men, and although I myself attended other members of the same family, well knowing them to suffer from lumbrici, a disease most common in this neighbourhood, especially amongst the children of the poor, and resulting apparently from their using, for domestic purposes, impure water.

With the above to me most evident symptoms of poisoning, I am constrained to believe that the course pursued was not only justifiable, but imperatively demanded, and that any other mode of treatment would have rendered me justly obnoxious to severe criticism and censure. This boy had never until now suffered from symptoms of clisease of the abdomen ; he was never suspected to be the subject of worms. Medical men are, $\mathrm{I}$ apprehend, alone responsible for using to the best of their judgment the evidences presented in each individual case; nor should I be prepared to infer the existence of serious disease in a child habitually helping his father at the plongh. The boy's condition shortly before the fatal seizure-the clean tongue, perspiring skin, quiet pulse of 80 per minute, and, above all, the abdomen less full than natural, and bearing very firm pressure on every part without eliciting complaint of pain or uneasiness--seemed to me to declare conclusively the $a b$ sence of idiopathic inflammation of the stomach, a thing almost tunheard of, or of any other abdominal viscus. I may mention that hernia was looked for while the child was vomiting, but none existed.

Watton Cottage, near Ware, Herts, August, 1857.

** We have been informed, that $\mathrm{Mr}$. Archer was subjected to some annoyance respecting this case, and that the course he had pursued gave rise, in the minds of the ignorant and unkindly disposed, to suspicions of improper practice on his part, too ridiculous to be $d$ welt upon. The facts of the case speak for themselves, and are a sufficient justification of the rectitude of intentions of the surgeon, and the perfect propriety of his mode of treatment. - SUB-ED. L.

\section{NOTES OF}

\section{CASES OF RUPTURED PERINAUM. WITH REMARKS.}

By I. BAKER BRoWN, EsQ., F.R.C.S. (by Exam.,) \&c., SURGRON-ACCOUCHEUR TO ST. MARY'S HOSPITAX.

THE object of the present paper is to extend the list of cases (thirty-two in number) which I placed before the profession some two years ago.

CASE 1.-L. F-, aged twenty; rupture of perinæum up 216 to, but not through the sphincter; three months' duration; operation ; cure.

Remarls.-Admitted into Boynton ward, under Mr. Baker Brown, on October 12th, 185\%. She has had one child, with which she was in labour from Sunday until Wednesday. Pro. lapsus of the uterus came on about a month after, which is a source of very great pain and discomfort to her.

Oct. 13th.-Usual operation.

16th.--Deep sutures removed.

18th. - Superficial sutures removed.

2lst. - Bowels moved.

Jan. 13th, 1856,-Discharged quite cured.

Remarks. - Although this case was not of the severest form of rupture, still the prolapsus of the womb rendered an opera. tion desirable.

Case 2.-A. C-, aged twenty-five; complete rupture through the sphincter; six weeks' duration; operation; cure.

Admitted into Boynton ward, under Mr. Baker Brown, on Nov. 6th, 1855. Was in labour with her first child forty-eight hours. When the bowels are confined she can retain her fæces for a time, but upon the slightest relaxation occurring, the fæces escape involuntary. A good deal of prolapsus of the uterus.

Nov. 7th.--Usual operation performed.

10th. - Deep sutures removed.

12th. - Superficial sutures removed.

19th. - Bowels moved.

30th. - Discharged quite cured.

Remarks. - This case being one of a severe form, it will be observed that it was cured, and the patient discharged in less than a month.

CASE 3.-A. M. B-, aged twenty-one; complete laceration through the sphincter; a month's duration; operation; cure.

Admitted into Boynton ward, under Mr. Baker Brown, Nov. 27 th, 1855. Six months ago was delivered of her first child after a tedious labour. At first could not retain her urine, but latterly has somewhat recovered that power, but not com. pletely. The fæees pass quite involuntarily whenever the bowels are at all relaxed. The uterus lower in the pelvis than normal.

Nov. 28th.-Usual operation performed.

30th. - Some ulceration in the track of the deep sutures, which were therefore removed.

Dec. 3rd.-Superficial sutures remored.

6th. - Bowels moved.

27 th. -Discharged quite cured.

Remarks. - This case is another of severe rupture, and yet cured, and the patient discharged in a month.

CASE 4.-M. D-.-, aged sixty-five; complete rupture through the sphincter; twenty years' duration; operation; fiilure.

Admitted into Boynton ward, under Mr. Baker Brown, on Dec. 3rd, 185̃5. Twenty years ago was delivered of her first child, a breech presentation. She could not retain her fæces. Eight years afterwards had her second child. T'welve months since the uterus became prolapsed, and has much increased her sufferings. She is in the habit of taking opium to relieve her sufferings; indeed she gets no sleep without it.

Dec. 3rd.-Usual operation performed.

Sth. - Ulceration in track of deep sutures, which were there. fore remeved; usual dose of opium takes no effect.

9th.-A good deal of bleeding from the track of one of the sutures.

10th.-Bowels moved.

Jan. 14th. -Discharged, with more power over the rectum.

Remarks. - The patient's health was so very bad, and her constitution so impaired, that I determined not to attempt a second operation. She had a slight attack of paraplegia in the hospital after the operation.

CASY 5.-A. M-, aged nineteen; complete laceration through the sphincter; sixteen months' duration; operation; cure. Admitted into Boynton ward, under Mr. Baker Brown, on May 17th, 1856. She was delivered of her first child with forceps sixteen months ago. She lost the entire control over her bowels, and went to St. George's Hospital, where she remained three weeks without receiving the least benefit.

May 21st. - Usual operation performed.

24th. - Deep sutures removed.

26th. - Superficial sutures removed.

June 5th. - Bowels moved.

14th. - Discharged perfectly cured.

Remarks. - This case wasalso oured, and the patient discharged in less than a month. 
CASE 6.-Mrs. B-_, aged forty-seven; complete laceration through the sphincter, thirty years' duration, with retracted anus, having been born without anus; operation; relieved.

Sent me by Dr. Locock. Thirty years ago was delivered of her first child. Slight control over her bowels. Bearing-clown pains very bad.

March 21st.--Performed the usual operation, assisted by Dr. Jones, Messrs. G. Brown, Moullin, and Laurence.

23rd.-Removed the deep sutures.

25th.--Removed the superficial sutures.

Remarks. - There was a great tendency to ulceration, which continued for some time, and destroyed a portion of the new perinæum. Finally, everything healed, and she left town in a much more comfortable state, with control over the bowels, and the os vagina so contracted that I could only pass a very small speculum. Still the bladder prolapsed somewhat, and will require a second operation for its cure. This case was a very bad one, from the retraction of the anus, which was at least two inches higher up than normal.

CASE 7.-Mrs. D-, aged thirty-six; complete laceration through the sphincter; operation; cure.

Mother of eleven children; at the birth of the tenth, the perinæum was ruptured, and she lost all control over the bowels. An operation was performed by Mr. Bradley, of Greenwich, in November, 1554. At the birth of her next child the parts again gave way, and she became as bad as before.

Jan. 26th, 1856. - Although in the third month of pregnancy I performed my usual operation, in the presence of Messrs.

Bradley, Laurence, and Mr. Arthur Brown

2Sth.-Removed the deep sutures.

30 th. - Removed the superficial sutures.

Feb. 4th. - Bowels moved.

20th. - Quite cured.

Remurks. - It will be observed that I operated on this case although the patient was presnant, being the third case in which I have done so successfully. I heard from Mr. Bradley in December that she had been confined of a large child; a small bit of the fonrchette gave way, to which Mr. Bradley applied an interrupted suture, and all healed in three days.

CASE 8.-Mrs. B-_ aged twenty-seven; complete laceration through the sphincter; two weeks' duration; operation; partial success ; second operation; cure.

In labour with her first child, and the forceps were applied to plave the head of the child in the right position. After they were removed, a sudden pain expelled the child, tearing through the perinæum and sphincter. The tear extended very far back through the septum. At the end of a fortnight I was consulted, and found nearly all the torn parts covered with new mucous membrane, so I was compelled to operate as in old standing cases.

Sept. 1lth.-Usual operation, but without chloroform.

14th-Removed the deep sutures; slight alceration had commenced.

26th.-Bowels moved; some fæecal matter escaped throngh a recto-vaginal opening.

29th. - External opening healed.

0ct. 17th. - The opening being still large, I again operated, being assisted by Dr. Frazer, and Miessrs. Andrews and Coventry.

30th. - Bowels moved.

Remarks. - On December Ist a most careful examination convinced me that the perinæum and septum were perfect and sound, and she continuing quite well up to this time. I wish to observe on this case, that the operation onght to have been performed at once; but a distinguished professor of midwifery having been called in, recommended waiting for eight days. I cannot understand his reasons, and protest against the practice.

CASE 9.-Mrs. T- of Birkenhead, aged thirty-five; complete laceration through the sphincter; eight years' duration; operation; cure.

During labour with her first child, eight years ago, forceps were used for her delivery, and a recto-vaginal opening produced. During labour with her second child, the remaining portion of the perineum gave way, and a complete laceration was produced. She lost entirely all control over her bowels.

Oct. 9th, 1856. - I went up to Birkenhead, and operated in the presence of, and assisted by, Drs. Walker and Dixon, and Messrs. Walker and Sutiers. She was in the second month of pregnancy.

1lth.-Dr. Walker removed the deep sutures; parts healed by first intention. 15th.- Removed the superficial sutures.

25th.--Bowels moved.

Remarks. - Nov. 20th: Dr, Walker reports the patient as quite well, with a very sound and perfect perinæum. This is another case where I operated, although the patient was pregnant.

CASE 10. - J. R-, aged thirty; laceration of the perineum extending through a portion of the sphincter; six hours' duration; operation; cure.

In labour with her first ohild for forty-eight hours. The forceps were applied, but before traction was made, a sudden. pain expelled the child, and tore through the perinæum. This occurred at seven A.M.

Ang. 24th. - I was immediately sent for, but being out of town, my friend and assistant, Mr. Philip Harper, went, and at three P.M. performed ny usual operation.

27 th. - Removed the deep sutures; ulceration had commenced.

31st. - Removed the superficial sutures.

Sept. 6th. - Bowels moved.

30 th. - Quite cured.

Remarks. - This case illustrates the practicability of operating immediately at the lesion. The patient was thereby saved a second confinement to bed and its attendant expense.

(To be concluded.)

REPORT OF A CASE OF

\section{DISLOCATION OF THE ANKLE,}

WITH

FRACTURE OF FIBULA, AND COMMINUTED FRACTURE OF TIBIA, FOLLOWED BY NECROSIS OF THE WHULE ASTRAGALUS.

By R. BARWELL, Esq., F.R.C.S., ASIISTA-ST-SURGEOA to THE CHARING-CROSS HOSPITAL.

Os the 27th of October I was asked to see Mrs. G-, in consultation with Mr. Creed, of Geeenwich. The patient is fifty-six years old, somewhat intemperate, and of very impatient temper. She is subject to bilious attacks, violent indigestions, and irregular purgings. The following account of the case previous to my seeing her is from the description of $\mathrm{Mr}$. Creed:-

On the 10th of October she was jumping from a height, and injured her foot, so that the above-named gentleman and his assistant, Mr. Haddlesey, were called. The foot was dislocated outward, the fibula being broken about an inch above the malleolus. The tibia had suffered comminuted fracture, the upper break being from three to four inches above the joint. The inner malleolus was broken off, and remained attached by its ligamentous connexion to the astragalus. The lower edge of the shaft of the tibia, which corresponded to this fracture, projected violently against the skin, and nearly broke throngh it. The patient was much excited and very impatient of control. The dislocation was with some difficulty reduced, and the limb put upon two latera? splints. She would not, however, remain quiet, but moved about, first to one side, then to the other; sometimes she took off the splints.

October 27 th. - When $\Upsilon$ saw her the leg was in two lateraI splints. She complained much of pain in the foot, and running up the tibia. On removing the splints, the inner side of the ankle was found red, puffy, and glazed. A wound, discharging pus and synovia, ran from before backwards the whole breadth of the inner malleolus immediately over the fracture. The foot was in very good position, but the lower end of the tibia projected inward; crepitus was distinct on twisting the foot laterally. The pulse was rather weak, about 100 ; appetite feeble. She had irregular purgings. Such, however, had been her condition for some weeks previous to the accident, and did. not therefore show so grave a disturbance as if it had been the result of the injury. I placed the limb on an outside splint, banclaging so that an uncovered part remained over the inner ankle for the application of poultices. The leg was then swung in a crudle in such a position as to allow the duscharge to gravitate out of the wound. She was to continue the sulphuric acid, which seemed to agree. Some strengthening diet was ordered and she was put upon a distinct allowance of her accustomed stimulus. 